\title{
Calibration of the Cepheid Distance Scale
}

\author{
Wolfgang P. Gieren ${ }^{1}$ and Pascal Fouqué ${ }^{1,2}$ \\ ${ }^{1}$ Astrophysics Group, Facultad de Física \\ Pontificia Universidad Católica de Chile, Santiago, Chile \\ ${ }^{2}$ Observatoire de Meudon, Paris, France
}

\begin{abstract}
The absolute calibration of the Cepheid period-luminosity (PL) relation with galactic Cepheids is discussed. Various methods, most importantly the cluster ZAMS-fitting scale and the Baade-Wesselink scale are found to yield PL zero points which agree within $\sim \pm 0.1 \mathrm{mag}$. The present Cepheid calibration sets the Large Magellanic Cloud at $\mu_{o}(L M C)=18.6 \pm 0.1 \mathrm{mag}$, in good agreement with the distance derived from SN 1987A and other methods except RR Lyrae stars which seem to give a shorter distance scale.
\end{abstract}

\section{Introduction}

Cepheid variables continue to be the most important calibrators of the extragalactic distance scale. This is so because they are intrinsically bright, easy to detect and obey a period-luminosity (PL) relation for which we have reasons to believe that it is universal.

In order to derive absolute distances to galaxies one has to calibrate the local distance scale using Cepheids in our Galaxy. This is a complicated problem and has occupied researchers for some 80 years now without full confidence in the results being attained even today.

Recent reviews dealing with Cepheids as standard candles have been given, among others, by Madore and Freedman (1991), Walker (1988), and Feast and Walker (1987). In the past few years, progress has been made in several respects. Improved Cepheid distances to a number of nearby galaxies have been measured using multicolor CCD photometry (reviewed elsewhere in this volume), and there has been important work on improving our knowledge of the absolute magnitudes of galactic Cepheids using a variety of methods, which will be reviewed here.

It is clear that Cepheid observations in infrared passbands have attained increasing importance in recent years for distance determinations of extragalactic Cepheids, mainly because of less problems with reddening corrections and reduced light amplitudes in the infrared. Still, work on the galactic calibrators has mainly involved observations in the $\mathrm{B}$ and $\mathrm{V}$ passbands, and for this reason this review will concentrate on the calibration of the $\mathrm{PL}(\mathrm{V})$ relation. 
Infrared Cepheid PL relations have been discussed elsewhere (e.g. Madore and Freedman 1991; Feast and Walker 1987; Laney and Stobie 1986).

\section{The metallicity dependence of the $P L$ and $P L C$ relations}

Theory predicts (e.g. Stothers 1988) that the slope of the PL(V) relation is practically metallicity independent. Observations of Cepheids in the Galaxy and the Magellanic Clouds, as well as in some Local Group galaxies seem to confirm this, yielding PL slopes close to -2.9. Different slopes which have occasionally been found can probably be attributed to selection effects due to the observations of only the brightest Cepheids in a galaxy which produces a bias of the Malmquist type (Sandage 1988), or to calibration errors in photographic photometry near the plate limit.

Theoretical work also shows that the zero point of the bolometric PL relation is metallicity independent (Stothers 1988): The bolometric correction BC is a weak function of metallicity (Laney and Stobie 1986) but since BC is very small in the $\mathrm{V}$ band no appreciable metallicity effect on the $\mathrm{PL}(\mathrm{V})$ zero point is expected. This has recently been confirmed by the work of Freedman and Madore (1990) on M31 Cepheids which has shown that there are no significant zero point shifts of the PL relations obeyed by Cepheids in fields of widely different radial distances from the center of M31 having metallicities ranging over a factor of $\sim 5$.

The basic problem in the use of a PLC relation for distance determinations is its strongly increased sensitivity to metallicity differences in the Cepheids (e.g. Stothers 1988). Caldwell and Coulson (1986) have found a PLC zero point offset of $0.51 \mathrm{mag}$ between our Galaxy (at $z=0.02$ ) and the SMC (at $z=0.005$ ). For galaxies for whose Cepheids accurate metallicities and reddenings are available the use of a PLC relation may be as good or even preferable to the use of a PL relation, but in practice this information is only available in the Magellanic Clouds. Another problem which is still with us is the true size of the color coefficient in the PLC relation which is difficult to determine due to the necessity for disentangling the effects of differential reddening. This is briefly discussed in Section 7 of this review.

\section{Calibration on the galactic Cepheid PL(V) relation}

There are at least four observational methods to find Cepheid absolute magnitudes. These are:

a) ZAMS-fitting to the color-magnitude diagrams of clusters containing Cepheids

b) Baade-Wesselink methods

c) Statistical parallaxes

d) Binary systems containing Cepheids

The cluster method has traditionally been considered as the most reliable means to obtain Cepheid luminosities. However, there are a number of severe difficulties with 
this method. There are few rich and nearby clusters containing Cepheids; most clusters are distant and sparse, and Cepheid membership is sometimes quite controversial due to the lack of additional membership information such as radial velocities of the cluster stars. Most clusters have high and variable reddening due to their low galactic latitudes, and have high field star densities contaminating the observed CMD's. The ZAMS is sensitive to the cluster metallicity (e.g. VandenBerg and Bridges 1984) and this is often not known, and there is the problem of the true distance of the standard cluster. The Pleiades are now generally used for this purpose rather than the Hyades cluster because of less problems with evolved stars on the upper main sequence due to its younger age, and of its solar metallicity. Since van Leeuwen's (1983) trigonometric parallax study a value of $\mu_{o}$ (Pleiades) $=5.57 \mathrm{mag}$ has been favored but more recent evidence (e.g. Gatewood et al. 1990; Feast 1991) suggests a slightly larger value of $\sim 5.70$ which carries directly over into the ZAMS-fitting Cepheid distance scale.

Baade-Wesselink (BW) methods have the advantage of yielding Cepheid luminosities which are independent of any of the other methods. BW distances can be found to any Cepheid having the necessary data (photometry and radial velocities) available allowing the PL relation to be calibrated with a large number of stars. Problems with uneven filling of the Cepheid instability strip and a very limited range in period and color of the calibrating Cepheids, as inherent in the cluster method, can be largely avoided in BW studies, but there are possibly severe sources of systematic error, mainly regarding the color indices appropriate to those studies.

The statistical parallax method has most recently been used by Wilson et al. (1991) to derive Cepheid luminosities. The resulting Cepheid magnitudes have relatively large errors but serve as a valuable check on the other methods.

Analyses of Cepheid companions detected in the ultraviolet by the IUE satellite have yielded absolute magnitudes of binary Cepheids in several cases (Evans 1991; Böhm-Vitense 1985) and a typical error in the best studied cases seems to be \pm 0.3 mag (see reviews by Evans and Böhm-Vitense in this volume). The binary method is valuable as an additional means to obtain luminosity information on Cepheids, but it is still quite limited regarding the number of studied cases and the accuracy of the results.

\section{The ZAMS-fitting Cepheid distance scale}

Recent calibrations of the galactic Cepheid PL relation using the ZAMS-fitting method have been given by Gieren and Fouqué (1992)(=GF), Turner (1992), Fernie (1992), Walker (1988), and Feast and Walker (1987). The coefficients of least-squares solutions to the respective data sets used by these authors are given in Table 1 . Since the slopes are all close to -2.90 and their mean value is $-2.903 \pm 0.019$, GF argue that $-2.90 \pm 0.02$ should be adopted as the appropriate slope of the PL(V) relation. Redetermining the PL zero points of the different data sets with the slopes forced to -2.90 then yields the following results: 


$\begin{array}{llll}\text { FW 87: } & -1.251 \pm 0.038 & \sigma=0.203 & \mathrm{~N}=28 \text { stars } \\ \text { Wa 88: } & -1.228 \pm 0.036 & \sigma=0.191 & \mathrm{~N}=28 \\ \text { Fe 92: } & -1.203 \pm 0.029 & \sigma=0.26 & \mathrm{~N}=28(+28 \delta \text { Sct }) \\ \text { Tu 92: } & -1.192 \pm 0.022 & \sigma=0.068 & \mathrm{~N}=10 \\ \text { GF 92: } & -1.292 \pm 0.049 & \sigma=0.275 & \mathrm{~N}=32\end{array}$

There seems to be a strong selection effect in the small Turner sample (10 stars) which further exhibits an unrealistically low dispersion. GF find a zero point of $1.204 \pm 0.077$ from their absolute magnitudes if they restrict their sample to the 10 Cepheids studied by Turner, in agreement with his result, but with a $\sigma=0.243$ comparable to other studies.

The GF study yields a PL zero point $\sim 0.07$ mag brighter than the other ZAMSfitting studies. This is essentially due to the fact that they use Cepheid color excesses as given in Fernie (1990) and determined from the Cepheids themselves, rather than calculating the Cepheid color excesses from the cluster OB star reddenings, as done in the other studies. Another improvement in the work of GF, besides of including the most recent data on true cluster distance moduli, is a homogeneous treatment of $R$, the ratio of total to selective absorption. GF use the values of $R$ for the clusters as given in the original references if these were measured; in other cases they standardize the cluster $R$ values to

$$
R=3.06+0.25\left(<B>_{0}-<V>_{0}\right)+0.05 E(O B)
$$

using $\langle B\rangle_{0}-\langle V\rangle_{0}=-0.2$. The coefficients in (1) are from Olson (1975) and the zero point is chosen so that the value of $R$ agrees with the mean value found for 51 galactic clusters by Turner (1976). All $\mu_{o}$ (cluster) values given in the original references were adjusted to the corrected $\mathrm{R}$ (cluster) values and to $\mu_{o}$ (Pleiades) $=$ 5.57. The values of $R$ appropriate to the Cepheids were also calculated from (1) but using the Cepheid intrinsic colors and the Fernie (1990) E(B-V) values. The GF calibrating data are reproduced in Table 2.

\section{The Baade-Wesselink distance scale}

The most recent and most complete BW study which seems to be representative of other $\mathrm{BW}$ work over the past few years using appropriate color indices and techniques (e.g. Coulson, Caldwell and Gieren 1986; Caccin et al. 1981) is that of Gieren, Barnes and Moffett (1992) (=GBM). GBM use the surface brightness version of the BW technique which employs (V-R) $J$ as the Cepheid surface brightness indicator. Using improved values for the slope (from Thompson's (1975) method) and zero point (from the Cepheid model atmosphere calculations of Hindsley and Bell (1989), and from Cepheid effective temperatures of Pel (1978) and the bolometric correction scale of VandenBerg and Bell (1985)) in the surface brightness relation

$$
F_{V} \equiv \log T_{e}+0.1 B C=b+m(V-R) 。
$$


GBM find $\left\langle M_{V}\right\rangle=-1.32-3.06 \log \mathrm{P}(\sigma=0.29 \mathrm{mag})$ from 100 galactic Cepheids, and $\left\langle M_{V}\right\rangle=-1.37-3.01 \log \mathrm{P}(\sigma=0.27 \mathrm{mag})$ from 79 Cepheids with distance determinations more accurate than 10 percent.

Table 1. Recent Calibrations of the galactic Cepheid PL(V) relation

$$
\left\langle M_{V}\right\rangle=\mathrm{a}+\mathrm{b} \log \mathrm{P}
$$

\begin{tabular}{|c|c|c|c|c|c|c|}
\hline Source & Method & $\mathbf{N}$ & $\mathbf{a}$ & b & Disp. & $<M_{V}>(0.8)$ \\
\hline Feast \& Walker 87 & ZAMS-fitting & 28 & -1.224 & -2.927 & 0.205 & -3.57 \\
\hline Walker 88 & ZAMS-fitting & 28 & -1.222 & -2.906 & 0.193 & -3.55 \\
\hline Fernie 92 & ZAMS-fitting & 28 & -1.203 & -2.902 & 0.260 & -3.52 \\
\hline Turner 92 & ZAMS-fitting & 10 & -1.153 & -2.939 & 0.070 & -3.50 \\
\hline Gieren \& Fouqué 92 & ZAMS-fitting & 32 & -1.281 & -2.911 & 0.280 & -3.61 \\
\hline GBM 92 & $\begin{array}{l}\text { BW (surface- } \\
\text { brightness) }\end{array}$ & 100 & -1.37 & -3.01 & 0.27 & -3.78 \\
\hline НВ 92 & $\begin{array}{l}\text { BW (model } \\
\text { atmosph.) }\end{array}$ & 23 & -1.33 & -3.11 & 0.25 & -3.82 \\
\hline BS 84 & $\mathbf{H} \beta$ & $2^{1}$ & & & & -3.59 \\
\hline Schmidt 91 & $\mathrm{H} \beta$ & & & & & -3.50 \\
\hline Wilson et al. 91 & $\begin{array}{l}\text { Dynamical } \\
\text { Parallaxes }\end{array}$ & & & & & $-3.46 \pm 0.34$ \\
\hline
\end{tabular}

${ }^{1} \mathrm{CS}$ Vel and TW Nor

\section{Comparison of PL results from different methods}

The relevant information for a comparison of results is given in Table 1. In the last column the $\left\langle M_{V}\right\rangle$ at $\log \mathrm{P}=0.8$ as resulting from the different methods are shown. The main conclusions are:

a) The BW method yields a PL zero point $\sim 0.15$ mag brighter than the cluster scale. However, the present cluster scale is tied to $\mu_{o}$ (Pleiades) $=5.57$ which is likely to be underestimated, as discussed before. The GBM BW zero point would further be $\sim 0.05$ mag fainter if they had used the same reddening treatment as GF in their ZAMS-fitting study, making the BW scale only $\sim 0.10 \mathrm{mag}$ brighter than the cluster scale. This is clearly within the present uncertainties of both methods and strongly suggests that neither of the two methods contains systematic errors in excess of $\sim \pm 0.10 \mathrm{mag}$. 
Table 2. Cluster Cepheid Data used in the calibration of Gieren \& Fouqué (1992)

\begin{tabular}{|c|c|c|c|c|c|c|c|c|c|}
\hline Cepheid & $\begin{array}{l}\text { Cluster or } \\
\text { association }\end{array}$ & $\mathbf{Q}$ & $\log P$ & $\mu_{0}$ & $\mathrm{R}(\mathrm{OB})$ & $E(O B)$ & $\mathbf{R}$ (cep) & $\mathrm{E}(\mathrm{cep})$ & $<M_{V}>$ \\
\hline SU Cas & & C & 0.2899 & 7.07 & 3.1 & 0.29 & 3.178 & 0.287 & -2.01 \\
\hline EV Set & NGC 6664 & B & 0.4901 & 10.88 & ass & 0.60 & 3.112 & 0.679 & -2.86 \\
\hline SZ Tau & NGC 1647 & A & 0.4982 & 8.68 & 3.09 & 0.31 & 3.212 & 0.294 & -3.09 \\
\hline QZ Nor & NGC 6067 & A & 0.5782 & 11.13 & ass & 0.35 & 3.232 & 0.276 & -3.16 \\
\hline alpha UMi & & C & 0.5990 & 5.19 & & & 3.210 & 0.000 & -3.22 \\
\hline CEb Cas & NGC 7790 & $\mathbf{A}$ & 0.6512 & 12.68 & ass & 0.54 & 3.220 & 0.597 & -3.61 \\
\hline CF Cas & NGC 7790 & $\mathbf{A}$ & 0.6880 & 12.68 & ass & 0.54 & 3.241 & 0.566 & -3.38 \\
\hline CEa Cas & NGC 7790 & A & 0.7111 & 12.68 & ass & 0.54 & 3.240 & 0.597 & -3.70 \\
\hline UY Per & $\begin{array}{l}\text { King } 4 \text { or } \\
\text { Czerny } 8\end{array}$ & B & 0.7296 & 11.70 & 3.1 & 0.99 & 3.246 & 0.919 & -3.34 \\
\hline CV Mon & $\begin{array}{l}\text { Anon van } \\
\text { den Bergh }\end{array}$ & $\mathbf{A}$ & 0.7307 & 11.22 & 3.09 & 0.77 & 3.241 & 0.714 & -3.23 \\
\hline V Cen & NGC 5662 & C & 0.7399 & 9.11 & 3.1 & 0.31 & 3.218 & 0.289 & -3.22 \\
\hline CS Vel & Ruprecht 79 & B & 0.7712 & 12.55 & ass & 0.794 & 3.22 & 0.847 & -3.58 \\
\hline V367 Sct & NGC 6649 & A & 0.7989 & 11.27 & ass & 1.35 & 3.251 & 1.284 & -3.89 \\
\hline BB Sgr & Collinder 394 & $\mathbf{C}$ & 0.8220 & 9.05 & 3.1 & 0.25 & 3.246 & 0.284 & -3.05 \\
\hline U Sgr & M25 & A & 0.8290 & 8.95 & & 0.48 & 3.260 & 0.403 & -3.57 \\
\hline DL Cas & NGC 129 & A & 0.9031 & 11.12 & 3.20 & 0.51 & 3.242 & 0.533 & -3.88 \\
\hline S Nor & NGC 6087 & A & 0.9892 & 9.79 & 3.1 & 0.19 & 3.259 & 0.189 & -3.99 \\
\hline Zeta Gem & & C & 1.0065 & 7.75 & & & 3.256 & 0.018 & -3.89 \\
\hline TW Nor & Lynga 6 & A & 1.0328 & 11.41 & ass & 1.34 & 3.292 & 1.338 & -4.14 \\
\hline V340 Nor & NGC 6067 & $\mathbf{A}$ & 1.0526 & 11.13 & ass & 0.35 & 3.288 & 0.315 & -3.80 \\
\hline VY Car & Ass Car OB2 & B & 1.2767 & 11.42 & 3.05 & 0.28 & 3.302 & 0.243 & -4.76 \\
\hline RU Sct & Trumpler 35 & B & 1.2945 & 11.61 & 3.0 & 1.03 & 3.276 & 0.957 & -5.26 \\
\hline RZ Vel & Ass Vel OB1 & B & 1.3096 & 11.24 & 2.88 & 0.35 & 3.275 & 0.335 & -5.25 \\
\hline WZ Sgr & C1814-191a & A & 1.3394 & 11.27 & 3.0 & 0.61 & 3.318 & 0.467 & -4.80 \\
\hline SW Vel & Ass Vel OB5 & B & 1.3700 & 12.00 & 3.20 & 0.38 & 3.278 & 0.349 & -5.02 \\
\hline T Mon & Ass Mon OB2 & B & 1.4317 & 11.02 & 3.2 & 0.20 & 3.310 & 0.209 & -5.59 \\
\hline KQ Sco & $\begin{array}{l}\text { Ass Sco } \\
O B \text { anon }\end{array}$ & B & 1.4578 & 12.32 & 3.04 & 1.00 & 3.365 & 0.896 & -5.52 \\
\hline U Car & Ass Car OB2 & B & 1.5889 & 11.42 & 3.05 & 0.34 & 3.298 & 0.283 & -6.07 \\
\hline RS Pup & & C & 1.6172 & 11.28 & & & 3.329 & 0.446 & -5.75 \\
\hline SV Vul & Ass Vul OB1 & B & 1.6532 & 11.82 & 3.0 & 0.49 & 3.309 & 0.570 & -6.46 \\
\hline GY Sge & Ass $O B$ anon & $\mathrm{B}$ & 1.7081 & 12.66 & 3.0 & 1.30 & 3.390 & 1.140 & -6.29 \\
\hline S Vul & Ass Vul OB2 & C & 1.8299 & 13.23 & 3.0 & 0.88 & 3.362 & 0.827 & -7.05 \\
\hline
\end{tabular}

Note: $\mathrm{Q}=$ quality. A: cluster membership well established. B: cluster membership more uncertain. C: Cepheid not in cluster or association or membership very uncertain. 
b) The results from $\mathrm{H} \beta$ photometry of cluster $\mathrm{B}$ stars agree with the ZAMS-fitting scale if the Balona and Shobbrook (1984) calibration is used and the TW Nor and CS Vel clusters are rejected because of their very low number of $B$ stars. In this case, $\left\langle\mu_{o}\left(H_{\beta}\right)-\mu_{o}(Z A M S)\right\rangle=-0.03 \pm 0.09$ mag from six clusters. Including TW Nor and CS Vel, the difference becomes $-0.20 \pm 0.13$ mag.

c) The model atmosphere results of Hindsley and Bell (1989), as corrected by Hindsley (1992; private communication) agree with the BW result of GBM.

d) Cepheid absolute magnitudes obtained from statistical parallaxes and binary companions agree, within their large uncertainties, with the other results.

The Cepheid distance scale according to the galactic calibration discussed here sets the Large Magellanic Cloud at $\mu_{\circ}(L M C)=18.6 \pm 0.1 \mathrm{mag}$. This is in excellent agreement with the LMC distance modulus found from the expansion parallax of supernova 1987A of $18.50 \pm 0.13$ (Panagia et al. 1991) and seems to be supported by all other distance indicators except RR Lyrae stars (Walker 1992; see review in this volume).

\section{A note on the color term in the PLC relation}

The PLC relation can be written as

$$
<M_{V}>=\alpha+\beta \log P+\gamma\left(<B>_{0}-<V>_{0}\right)
$$

To avoid correlated errors in the solution of (3), we write

$$
<V>-\mu_{o}=\alpha+\beta \log P+\gamma(<B>-<V>)+\delta E(B-V)
$$

where $\mu_{0}=\langle V\rangle_{0}-\left\langle M_{V}\right\rangle$ and $\gamma+\delta=\mathrm{R}$ (ratio of total to selective absorption).

We have solved for (4) using the GAUSSFIT routine of W.H. Jefferys of Austin and the 100 Cepheid sample of GBM, assuming $\sigma(\log P)=0, \sigma(B-V)=\sigma(V)=0.02$ for all stars, taking individual $\sigma_{i}(E(B-V))$ from Fernie (1990) and $\sigma_{i}\left(\mu_{o}\right)$ from GBM, and taking $R=3.3$. This yields

$$
<M_{V}>=-1.45-2.96 \log P+0.04\left(<B>_{0}-<V>_{0}\right)
$$

which is not different from the GBM PL relation $\left\langle M_{V}\right\rangle=-1.37-3.01 \log P$. This result shows that no significant color term can be extracted from the galactic Cepheid data, probably due to the relatively large errors in the individual Cepheid distances.

Walker (1988) gives for the LMC PLC relation, corrected to solar metallicity

$$
<M_{V}>=-2.11-3.53 \log P+2.13\left(<B>_{0}-<V>_{0}\right)
$$

Using a purely statistical approach and assuming that the extra scatter observed in the LMC period-color relation, as compared to the galactic PC relation $(\sim 0.11$ 
mag in the LMC (e.g. Martin, Warren and Feast 1979) as compared to $\sim 0.07$ mag in the Galaxy (GBM) is solely due to larger uncertainties in the determination of color excesses for LMC Cepheids than for their galactic counterparts, Fouqué and Gieren (1992) have shown that the true color coefficient in (6) adopts a value of $\sim 0.7$. This is an interesting finding but it seems to be difficult to reconcile it with the spread in Cepheid magnitudes at a given period, as observed in LMC clusters rich in Cepheid variables (Welch 1992; see review in this volume). It shows, however, that we can still not be completely confident in the true value of the color coefficient of the PLC relation.

We are grateful to those astronomers who have sent data, comments and preprints during the preparation of this work. A grant of the Canadian Institute of Theoretical Astrophysics to WPG is gratefully acknowledged. We thank Dr. W.H. Jefferys for sending us his Gaussfit program and Gisela Hertling for doing part of the calculations of this work.

\section{References:}

Balona, L.A. and Shobbrook, R.R., 1984, M.N.R.A.S., 211, 375.

Böhm-Vitense, E., 1985, Ap.J., 296, 169.

Caccin, B., Onnembo, A., Russo, G. and Sollazzo, C., 1981, Astr. Ap., 97, 104.

Caldwell, Z.A.R. and Coulson, I.M., 1986, M.N.R.A.S., 218, 223.

Coulson, I.M., Caldwell, Z.A.R. and Gieren, W.P., 1986, Ap.J., 303, 273.

Evans, N.R., 1991, Ap.J., 372, 597.

Feast, M.W., 1991, SAAO Preprint 709. v

Feast, M.W. and Walker, A.R., 1987, Ann. Rev. Astr. Ap., 25, 345.

Fernie, J.D., 1990, Ap.J. Suppl., 72, 153.

Fernie, J.D., 1992, A.J., 103, 1647.

Fouqué, P. and Gieren, W.P., 1992, in preparation.

Freedman, W.L. and Madore, B.F., 1990, Ap.J., 365, 186.

Gatewood, G., Castelaz, M., Han, I., Persinger, T., Stein, J., Stephenson, B. and Tangreu, W., 1990, Ap.J., 364, 114.

Gieren, W.P. and Fouqué, P., 1992, A.J., submitted.

Gieren, W.P., Barnes, T.G. and Moffett, R.J., 1992, Ap.J., submitted.

Hindsley, R.B. and Bell, R.A., 1989, Ap.J., 341, 1004.

Laney, C.D. and Stobie, R.S., 1986 M.N.R.A.S., 222, 449.

Madore, B.F. and Freedman, W.L., 1991, Pub. A.S.P., 103, 933.

Martin, W.L., Warren, P.R. and Feast, M.W., 1979, M.N.R.A.S., 188, 139.

Olson, B.I., 1975, Pub. A.S.P., 87, 349.

Panagia, N., Gilmozzi, R., Macchetto, F., Adorf, H.M. and Kirshner, R.P., 1991, Ap.J., 380, L23. 
Pel, J.W., 1978, Astr. Ap., $62,75$.

Sandage, A., 1988, Pub. A.S.P., 100, 935.

Stothers, R.B., 1988, Ap.J., 329, 712.

Thompson, R.J., 1975, M.N.R.A.S., 172, 455.

Turner, D.G., 1992, private communication.

Turner, D.G., 1976, A.J., 81, 1125.

VandenBerg, D.A. and Bridges, R.B., 1984, Ap.J., 278, 679.

VandenBerg, D.A. and Bell, R.A., 1985, Ap.J. Suppl., 58, 561.

van Leeuwen, F., 1983, Ph.D. Thesis, Leiden University, Netherlands.

Walker, A.R., 1988, in The Extragalactic Distance Scale, ed. S. van den Bergh \& C.J. Pritchet ASP Confer. Series, Vol. 4, p. 89.

Wilson, T.D., Barnes, T.G., Hawley, S.L. and Jefferys, W.H., 1991, Ap.J., 378, 708.

\section{Discussion}

A. SANDAGE: I do not understand why there is any question about the value of the color term in the PLC relation. Its value is one of the best determined numbers in the relation because it depends only on the slope of the lines of constant period in the HR diagram. The only way the color term can be zero is if the lines of constant period are flat in the HR diagram which is impossible. I have always believed one must fix the color coefficient from the pulsation equation rather than attempting to determine it empirically.

W. P. GIEREN: I fully agree that the slope of the lines of constant period in the $\mathrm{HR}$ diagram cannot be zero, so there must be a color term in the PLC relation. However, there are uncertainties in the theoretical and semiempirical steps needed to calculate the color term which seem larger to me than usually assumed, and so there is a question if the true value is not significantly different from $\sim 2.5$. One empirical way to better clarify the question is to look at LMC clusters which contain lots of Cepheids of very similar period.

D. TURNER: I have a comment regarding the PL relation for cluster and association Cepheids, which seems to exhibit rather large scatter at the low period end. It is my belief that at least four of these Cepheids are overtone pulsators, so it makes a difference which period they are plotted for.

W. P. GIEREN: It is certainly possible that there are overtone pulsators in the sample, but the existing evidence is not convincing to me except for SU Cas. Anyway, even if one adopts all four shortest period Cepheids as overtone pulsators this does not make a significant difference in the PL coefficients although the scatter maybe somewhat lower.

D. WELCH: I have three comments. First, it is worth pointing out that the dominant error in most extragalactic distances is still the galactic calibration. Second, if most of the errors are with reddening, work at longer wavelengths. Third, the angular diameter situation should improve dramatically with SUSI coming into existence. 